\title{
Ultrafast photoinduced electron transfer in coumarin 343 sensitized $\mathrm{TiO}_{2}$-colloidal solution
}

\author{
Josef Wachtveitl, ${ }^{1 \dagger}$ Robert Huber, ${ }^{1}$ Sebastian Spörlein, ${ }^{1}$ \\ Jacques E. Moser, ${ }^{2 \ddagger}$ and Michael Grätzel ${ }^{2}$ \\ ${ }^{1}$ Institut für Medizinische Optik, Oettingenstr. 67, Ludwig-Maximilians-Universität \\ München, 80538 München, Germany \\ ${ }^{2}$ Laboratory for Photonics \& Interfaces, Department of Chemistry, Ecole \\ Polytechnique Fédérale de Lausanne, CH-1015 Lausanne, Switzerland
}

\begin{abstract}
Photoinduced electron transfer from organic dye molecules to semiconductor nanoparticles is the first and most important reaction step for the mechanism in the so called "wet solar cells" [1]. The time scale between the photoexcitation of the dye and the electron injection into the conduction band of the semiconductor colloid varies from a few tens of femtoseconds to nanoseconds, depending on the specific electron transfer parameters of the system, e.g., electronic coupling or free energy values of donor and acceptor molecules [2-10]. We show that visible pump/ white light probe is a very efficient tool to investigate the electron injection reaction allowing to observe simultaneously the relaxation of the excited dye, the injection process of the electron, the cooling of the injected electron and the charge recombination reaction.
\end{abstract}

\section{INTRODUCTION}

Photoinduced interfacial electron transfer in dye sensitized colloidal semiconductors is a process of fundamental importance. For example, the operation of photo-electrochemical devices with high conversion efficiency is based on that principle [1]. A high quantum yield is only obtained, if the reaction rate for charge separation is much higher than the rates of all other competing reactions (e.g., radiative and non-radiative deactivation processes). Therefore, it is of crucial importance that charge injection occurs on an ultrafast timescale. Electron transfer reactions on the femtosecond timescale for dye/ $\mathrm{TiO}_{2}$ systems have been observed recently by ultrafast laser spectroscopy [2-4, 7-11]. Near/mid infrared absorption and fluorescence upconversion measurements have been performed by several groups on dye $/ \mathrm{TiO}_{2}$ systems $[4,8]$. Here we report femtosecond time resolved transient absorption spectra over a wide spectral range covering the near UV and visible region with a high temporal resolution using a white light continuum as probing light in an excite and probe experiment. In contrast to fluorescence upconversion techniques, where only the population density of the photoexcited state of the dye can be probed, transient absorption measurements allow to distinguish between electron transfer and competing quenching mechanisms, e.g., intermolecular energy transfer between sensitizer molecules. So it also provides necessary additional information compared to near- or mid-IR investigations, where only the electron in the conduction band of the semiconductor can be probed. In this study we present data for the laser dye coumarin 343 adsorbed on the surface of $\mathrm{TiO}_{2}$ nanocrystals (Figure 1 , inset). We are able to assign the various spectral

†Josef.Wachtveitl@physik.uni-muenchen.de

łje.moser@epfl.ch components to ground state bleaching, cation formation and absorption of the electron in the conduction band and report fast interfacial electron transfer on a time scale faster than $100 \mathrm{fs}$. The experimental setup used for our investigations is described in $[12,13]$.

\section{EXPERIMENTAL METHODS}

Coumarin 343 was used as obtained by Kodak. Solutions of colloidal $\mathrm{TiO}_{2}$ were prepared as described in [5]. The absorption spectrum of the dye was only slightly affected upon adsorption on the $\mathrm{TiO}_{2}$ surface (Figure 1). The sensitized colloidal solution was circulated through a cuvette during the measurement, to prevent accumulation of photoproducts.

For the transient absorption measurements we use a home built $20 \mathrm{~Hz}$ Ti:sapphire laser system with a pulse width of about $100 \mathrm{fs}$ (FWHM), a pulse energy of $600 \mu \mathrm{J}$ and a central wavelength of $870 \mathrm{~nm}$. The main part of the energy is used for second harmonic generation to provide pulses at a wavelength of $435 \mathrm{~nm}$. These pulses were directly used to excite the coumarin and the coumarin $/ \mathrm{TiO}_{2}$ samples.

Recently, spectrally tunable excitation pulses could be obtained by pumping a nonlinear-opticalparametric-amplifier (NOPA) with the $435 \mathrm{~nm}$ pulses, that allow investigations of dye molecules in a broad spectral range (e.g., alizarin/ $\mathrm{TiO}_{2}$ with a maximum absorption at $495 \mathrm{~nm}$ ). Furthermore, the pulses generated this way can be compressed down to less than $20 \mathrm{fs}$ and provide the experimental basis to observe the fast electron injection processes directly [14].

A white light continuum for the probing branch of the setup is generated by focussing the $870 \mathrm{~nm}$ or the $435 \mathrm{~nm}$ light pulses into a $2 \mathrm{~mm}$ sapphire plate. The continuum generated with $435 \mathrm{~nm}$ light pulses covers a spectral range from $320 \mathrm{~nm}$ to $580 \mathrm{~nm}$, the one 


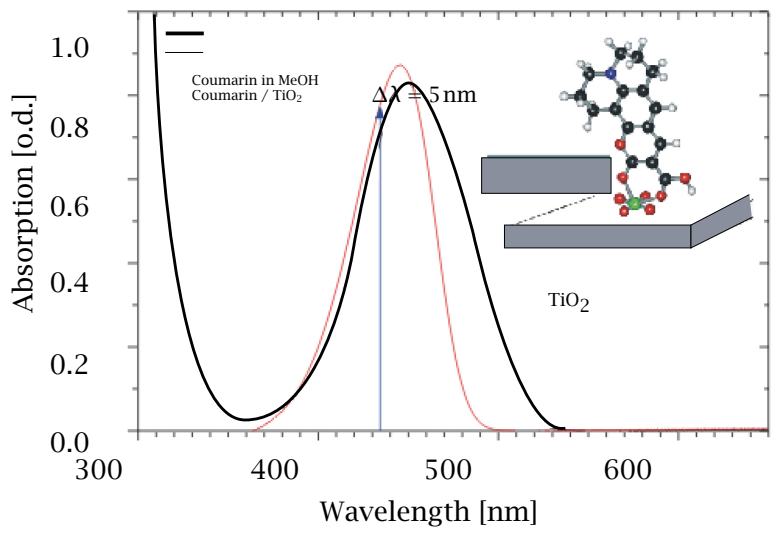

Figure 1. Absorption spectra of coumarin 343 in solution (methanol) and adsorbed to the surface of $\mathrm{TiO}_{2}$ nanoparticles. The coupling process induces only a small shift of about $5 \mathrm{~nm}$ in the main absorption band of the two samples. The excitation wavelength is indicated by the arrow. The strong increase in the absorption for the coumarin/ $/ \mathrm{TiO}_{2}$ sample for wavelengths $\lambda<350 \mathrm{~nm}$ reflects interband transitions from the valence- to the conduction band of the colloid. The arrangement of the coupled coumarin is shown in the inset.

generated at $870 \mathrm{~nm}$ extends from $450 \mathrm{~nm}$ to $1100 \mathrm{~nm}$. A spectral interval of about $10 \mathrm{~nm}$ around the generating wavelength of the white light can not be used due to temporal energy fluctuations. In order to prevent pulse lengthening by increasing chirp only mirror optics is used.

The white light is split into two parts, one is used to monitor the reference energy distribution of the white light, the other to probe the sample. The two parts are detected by two independent spectrometers each with a 42 channel array of photodiodes. The arrays are read out with a computer working in single shot detection mode. The absorption is defined by the ratio of sample and reference intensity. To calculate the absolute value for the absorption change every fifth pump pulse is blocked by a shutter and the absorption of the nonexcited sample is recorded. The continuous control of the absorption without excitation allows the detection of long term drift effects of the laser or degradation of the sample. This setup provides a high signal to noise ratio. Throughout the entire investigated spectral range the width (FWHM) of the cross correlation function was less than $110 \mathrm{fs}$ for the $435 \mathrm{~nm}$ pump pulses, less then $85 \mathrm{fs}$ for the excitation pulses around $500 \mathrm{~nm}$ generated with the NOPA. The polarizations of the pump and probe pulses were set to the magic angle.

\section{RESULTS AND DISCUSSION}

In the femtosecond time resolved experiments shown in Figure 2 transient absorption changes of coumarin 343 in solution are compared with coumarin 343 adsorbed on the $\mathrm{TiO}_{2}$ surface. The transient absorption changes in coumarin 343 are dominated by long lived excited state absorption and ground state bleaching. The absorption of the excited state can be observed at wavelengths $<400 \mathrm{~nm}$ while the bleaching of the ground state reflects the signature of the main absorption band $\left(\lambda_{\max }=445 \mathrm{~nm}\right)$ (Figure 1$)$. The characteristic absorption changes persist throughout the entire investigated temporal range ( $>3 \mathrm{~ns}$ ).

In contrast to the dye in solution, the coupled coumarin $343 / \mathrm{TiO}_{2}$ system shows an additional multiexponential decay of the signal with a dominating fast component of $350 \mathrm{fs}$ present in all transients recorded (Figures 2, 3, 4). A signal remains at long delay times, which resembles the characteristics of the difference spectrum for the charge separated system, stable over many orders of magnitude (Figures 3, 4). Figure 3 also shows the assignment of the various features of the difference spectrum: cation absorption $(\lambda<400 \mathrm{~nm})$, ground state bleaching $(400 \mathrm{~nm}<\lambda<520 \mathrm{~nm})$ and a weak, spectrally broad contribution of the electron in the conduction band of $\mathrm{TiO}_{2}(\lambda>520 \mathrm{~nm})$. Due to the similarity of the excited state absorption-derived from experiments on the dye in solution-and the cation band of coumarin/ $/ \mathrm{TiO}_{2}$, it is not possible to determine the dynamics of the electron injection process in this spectral region unambiguously.

Therefore, two possible mechanisms can explain the recorded kinetics:

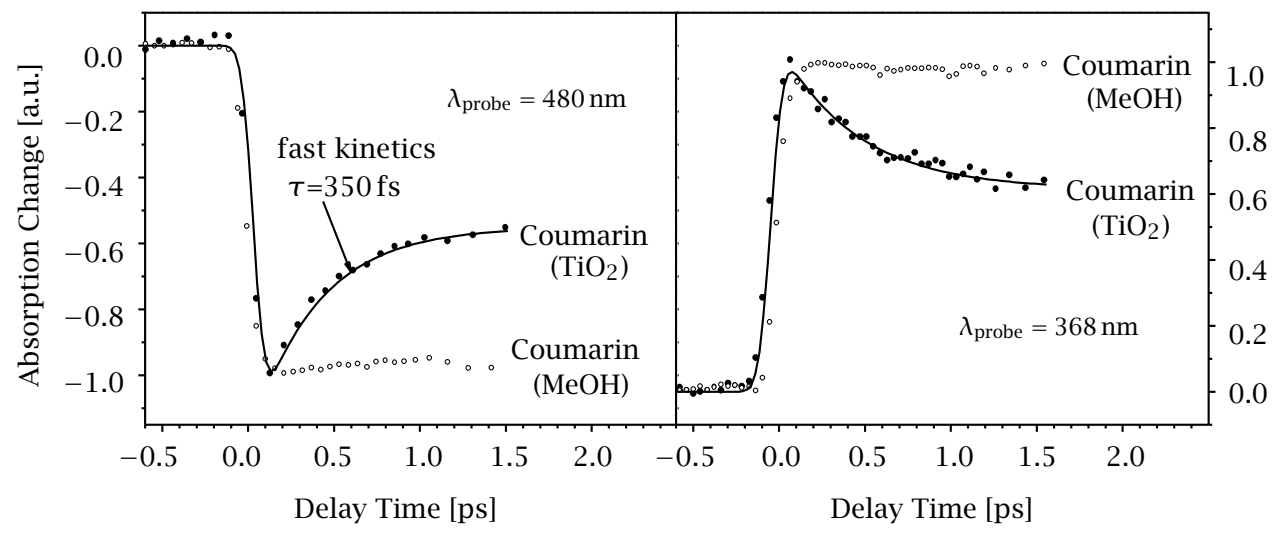

Figure 2. Femtosecond time resolved kinetics of coumarin 343 for uncoupled dye and dye/ $\mathrm{TiO}_{2}$ samples at two different probing wavelengths. 


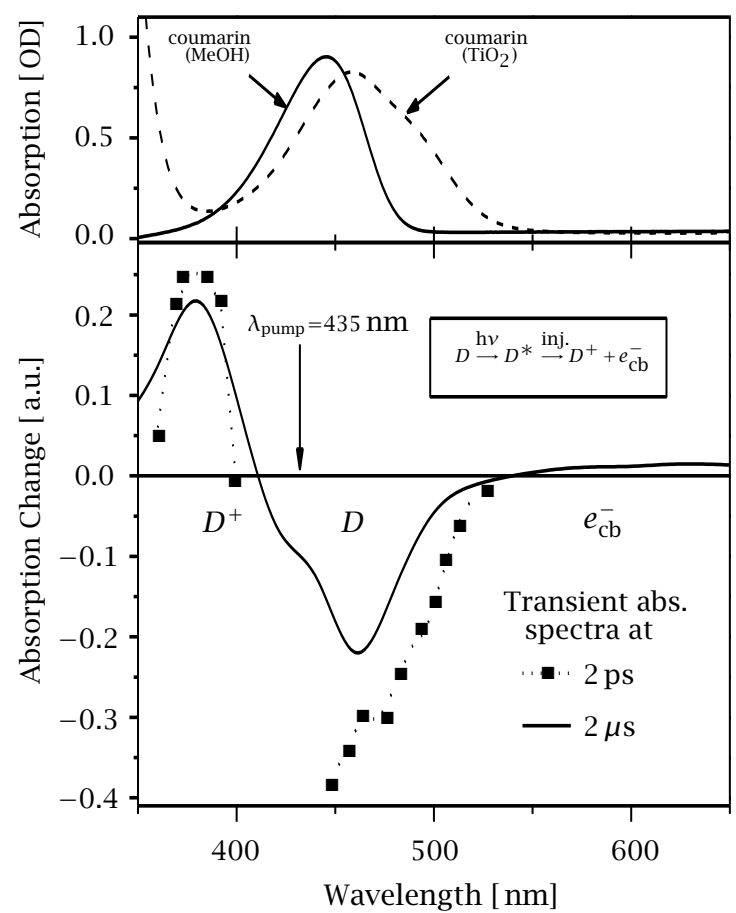

Figure 3. Top: Absorption spectra of coumarin 343 in methanol (-) and adsorbed onto the surface of nanocrystalline $\mathrm{TiO}_{2}$ (- - -). Bottom: Transient absorption spectra of coumarin 343 adsorbed on the surface of $\mathrm{TiO}_{2}$ nanocrystals after two different delay times and band assignment $\left(D^{+}\right.$: cation radical, $e_{\mathrm{cb}}^{-}$: conduction band electron) [6].

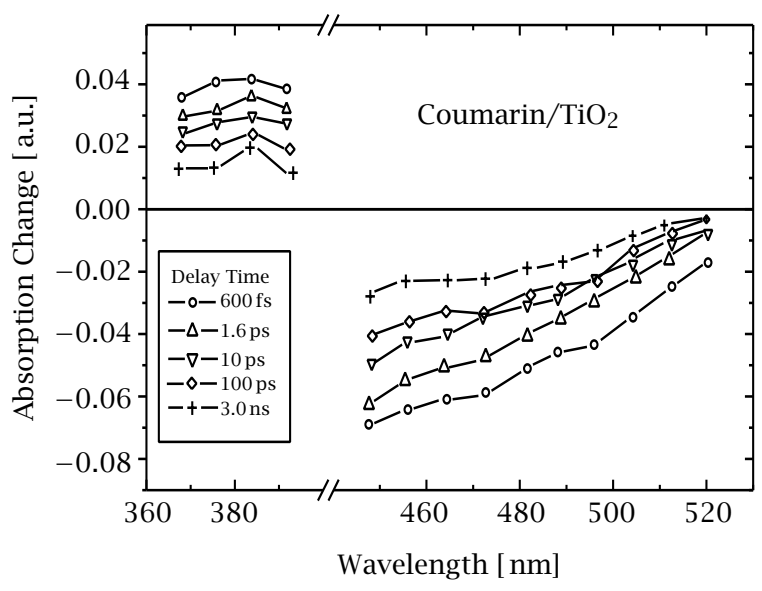

Figure 4. Transient spectra of coumarin $343 / \mathrm{TiO}_{2}$ at various delay times.

(i) The electron is injected with a time constant of $350 \mathrm{fs}$, indicated by the decay of the excited state and the formation of the coumarin cation radical signal. The signal decrease with a time constant of $\tau=350 \mathrm{fs}$ at $\lambda_{\text {probe }}=480 \mathrm{~nm}$ superimposed to the instantaneous bleaching of the ground state band is then caused by a contribution of photoinduced $D^{+}$molecules.

(ii) The electron is injected on a much shorter time scale ( $\tau<100 \mathrm{fs}$ ), but the detection of this fast com- ponent is hindered by the spectral overlap of the educt and the product states. Then the observed dynamics reflects charge recombination leading to a partial decay of the cation and the ground state features. This mechanism is supported by the lack of spectral evolution in the transient spectra at different delay times (Figure 4) and by measurements of ultrafast injection times in the range from $20 \mathrm{fs}$ to $180 \mathrm{fs}$ reported for this system by other groups $[4,8]$.

Measurements in the long wavelength region $(\lambda>$ $520 \mathrm{~nm}$ ) will allow to overcome this ambiguity, since a direct observation of the injected electron is possible. Also an improved time resolution of the setup using NOPAs is necessary for the reliable detection of the electron transfer reaction. These experiments are currently underway.

\section{CONCLUSIONS}

Ultrafast interfacial electron injection on the femtosecond time scale is observed from an electron donating dye molecule attached to the $\mathrm{TiO}_{2}$ surface. These results allow further systematic analysis of important electron transfer parameters. The influence of surface, solvent or donor-acceptor distance and effects like redistribution of vibrational excess energy or hot electron injection can now be addressed.

\section{REFERENCES}

[1] B. O’Regan and M. Grätzel, Nature 353 (1991), 737.

[2] Y. Tachibana, J. E. Moser, M. Grätzel, and J. R. Klug, D. R.and Durrant, J. Phys. Chem. 100 (1996), 20056.

[3] B. Burfeindt, T. Hannappel, W. Storck, and F. Willig, J. Phys. Chem. 100 (1996), 16463.

[4] J. M. Rehm, G. L. McLendon, Y. Nagasawa, K. Yoshihara, J. Moser, and M. Grätzel, J. Phys. Chem. 100 (1996), 9577.

[5] M. K. Nazeeruddin, P. Liska, J. E. Moser, N. Vlachopoulos, and M. Grätzel, Helv. Chim. Acta 73 (1990), 1788.

[6] J. E. Moser and M. Grätzel, Chem. Phys. 176 (1993), 493.

[7] R. Jimenez, G. R. Fleming, P. V. Kumar, and M. Maroncelli, Nature 369 (1994), 471.

[8] H. N. Gosh, J. B. Ashury, and T. Lian, J. Phys. Chem. 102 (1998), 6482.

[9] I. Martini, J. Hodak, and G. V. Hartland, J. Chem. Phys. 107 (1997), 19.

[10] M. Hilgendorff and V. Sundström, J. Phys. Chem. 102 (1998), 10505.

[11] T. Heimer and E. J. Heilweil, J. Phys. Chem. 101 (1997), 10990.

[12] T. Nägele, R. Hoche, W. Zinth, and J. Wachtveitl, Chem. Phys. Lett. 272 (1997), 489.

[13] M. Seel, E. Wildermuth, and W. Zinth, Meas. Sci. Technol. 8 (1997), 449.

[14] T. Wilhelm, J. Piel, and E. Riedle, Opt. Lett. 22 (1997), 1474. 


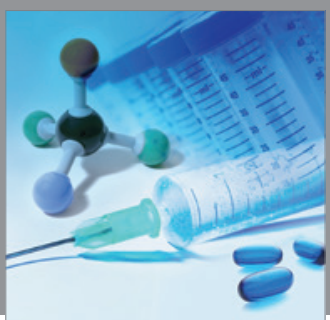

International Journal of

Medicinal Chemistry

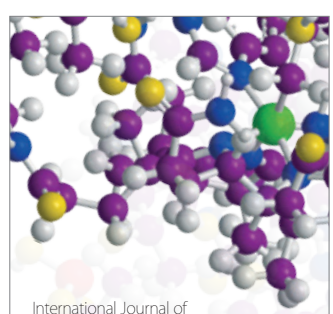

Carbohydrate Chemistry

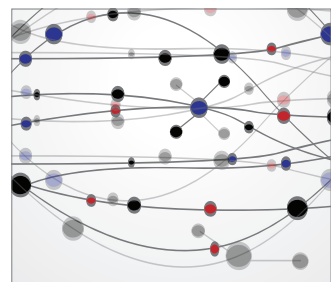

The Scientific World Journal
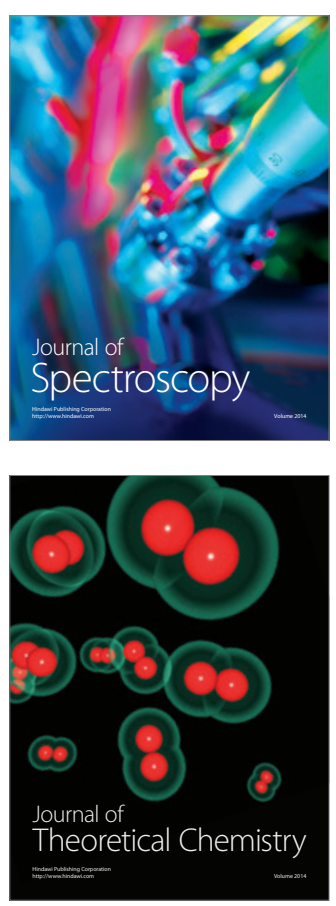
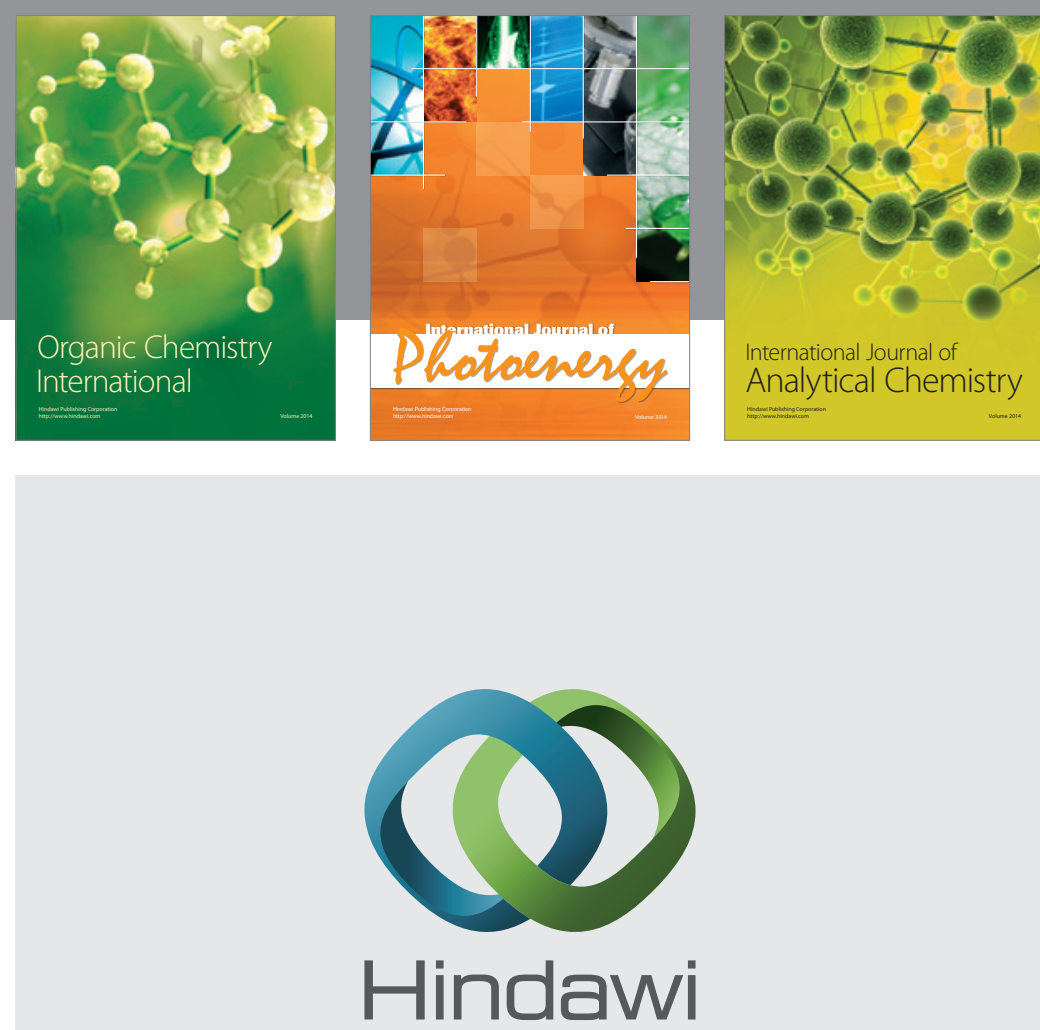

Submit your manuscripts at

http://www.hindawi.com
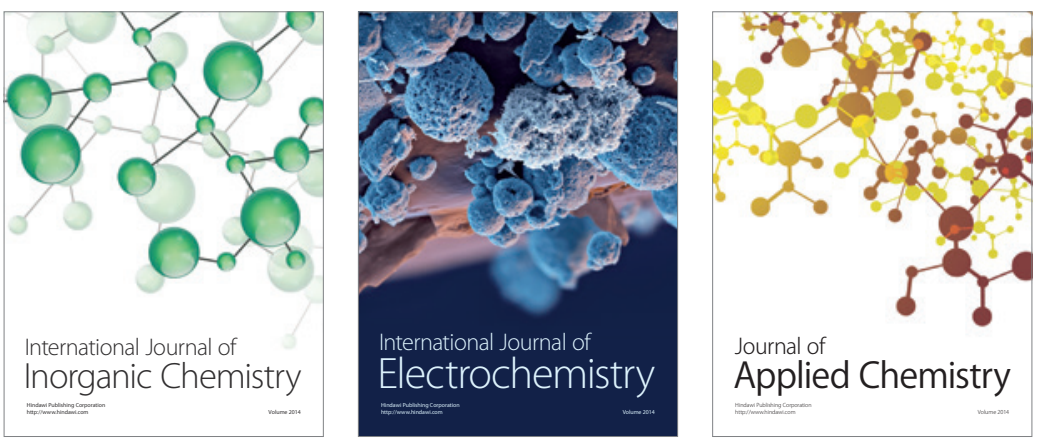

Journal of

Applied Chemistry
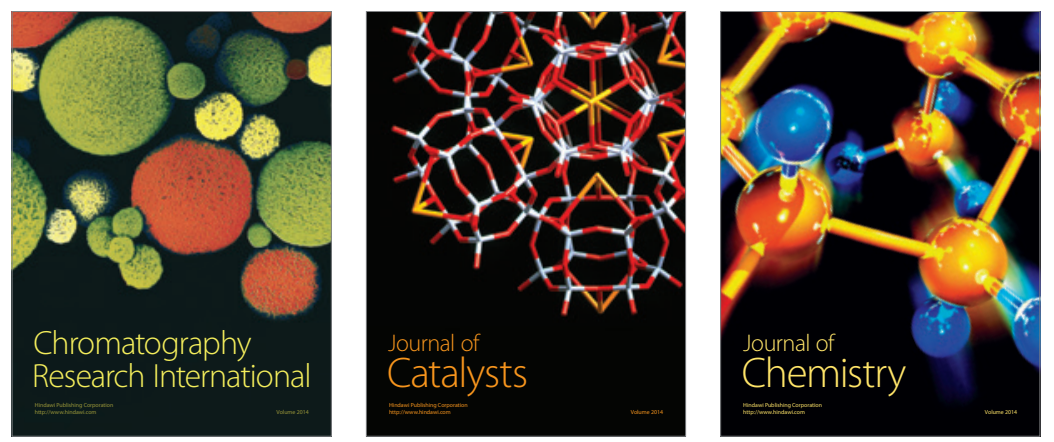
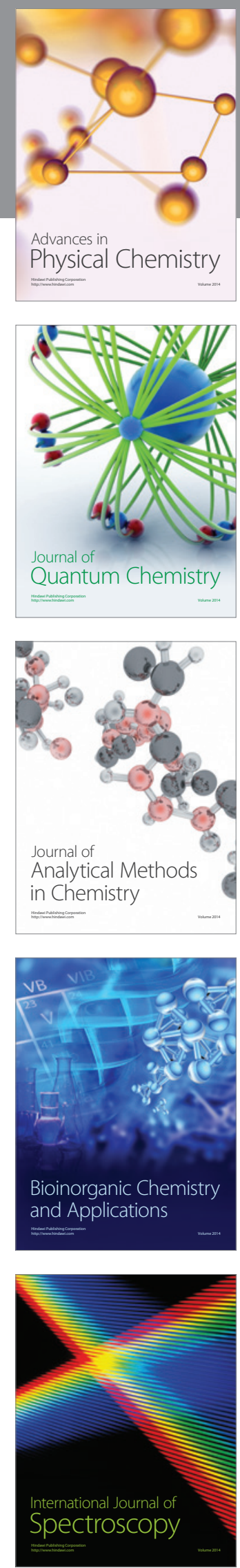